\title{
Multi-step-ahead, Short-Term Prediction of Wind Speed Using a Fusion Approach
}

\author{
Julian L. Cardenas-Barrera ${ }^{1}$, Eduardo Castillo-Guerra ${ }^{2}$, \\ Julian Meng ${ }^{2}$, and Liuchen Chang ${ }^{2}$ \\ ${ }^{1}$ Center for Studies on Electronics and Information Technologies, \\ Universidad Central "Marta Abreu" de Las Villas. Santa Clara. V.C. Cuba. \\ ${ }^{2}$ Department of Electrical and Computing Engineering. University of New Brunswick \\ Fredericton. N.B. Canada. \\ julian@uclv.edu.cu, \{ecastil,jmeng, lchang\}@unb.ca
}

\begin{abstract}
Wind power generation is a green solution to power generation that is receiving increasing interest worldwide. Wind speed forecasting is critical for this technology to succeed and remains today as a challenge to the research community. This paper presents a neural network fusion approach to multi-stepahead, short-term forecasting of wind speed time-series. Wind speed forecasts are generated using a bank of neural networks that combine predictions from three different forecasters. The wind speed forecasters include a naïve model; a physical model and a custom designed artificial neural network model. Data used in the experiments are telemetric measurements of weather variables from wind farms in Eastern Canada, covering the period from November 2011 to October 2012. Our results show that the combination of three different forecasters leads to substantial performance improvements over recommended reference models.
\end{abstract}

Keywords: Short-term wind speed forecasting, artificial neural networks, forecast combination.

\section{Introduction}

Wind power generation is a green and cost-effective solution to power generation, which has grown substantially worldwide. It is among the most competitive renewable technologies, projected to account for significant shares of the global power market in the near future [1]. However, the inherent variability and uncertainty of wind can lead to unexpected and sometimes substantial mismatches between scheduled and actual wind power [2]. This fact has driven the search for improved wind power forecasting methods [1-8].

Forecasting means that future values of wind power or speed $a(t+k)$ will be estimated as $\hat{a}(t+k \mid t)$ by some function $\varphi$, given some predicting variables $X(t)$ up to time $t$. A forecasting error $\varepsilon(t+k \mid t)$ is introduced and expected to be bounded. The target time $(t+k)$ is called the forecasting horizon.

$$
\hat{a}(t+k \mid t)=\varphi(X(t))
$$




$$
a(t+k)=\hat{a}(t+k \mid t)+\varepsilon(t+k \mid t),
$$

Forecasting horizons for wind power integration and operation span time frames ranging from a few seconds to weeks ahead [8]. Accurate short-term forecasts are critical for effective power management and receive considerable research attention $[1,7,9-13]$. Forecasting methods typically work better when the prediction is focused on specific time frames. Complex physical models for example, use numeric weather prediction (NWP) and are known to have good performance for horizons beyond 3-6 hours ahead [10]. The simplest model called persistence offers accurate wind speed forecasts for immediate horizons, typically up to around one hour ahead but also depending on weather conditions, can be reliable for up to 6 hours $[9,10]$. Statistical and machine learning approaches on the other hand, can generate reasonable forecasts for less than a day horizon $[2,8]$.

Forecast combination offers a way to reduce forecasting errors by combining the predictions from a number of forecasters [14-16]. Prediction gains are obtained by exploiting diversification if individual forecasters use different models of the underlying process they predict [17]. Combination success depends on how well mixing weights can be assigned to individual forecasters. This method is often preferred over finding one single best model. For wind speed forecasting, statistical and machine learning models are known to automatically reduce systematical errors due to their adaptation to the location of the wind farm; however as opposed to NWP, they have difficulties predicting rare atmospheric conditions $[6,8,11]$. Combining NWP based, statistical and machine learning models could help at getting the most of all methods.

This paper exploits this approach proposing a fusion method for multi-step-ahead, short-term (up to 6 hours ahead) prediction of wind speed. Neural networks (NN) are used to determine the best nonlinear combination of three predictors at different time leads. The predicting models include persistence, artificial neural network and NWPbased predictors. Performance is assessed using recommended error measures and reference models $[1,9,10]$.

\section{Case Study and Data Description}

The data used in this study come from one-year-long recordings of several measured weather variables at four on-shore wind farms in Eastern Canada. The wind farms are named herein as WindFarm-1, WindFarm-2, WindFarm-3 and WindFarm-4. The observed variables include temperature, wind speed, and wind direction measured at the hub heights ( 80 meters), every five minutes. Table 1 shows mean wind speeds,

Table 1. Wind characteristics during the period under study

\begin{tabular}{lccc}
\hline Wind farm & Mean Wind Speed (scale, shape of Weibull dist.) & Wind Direction \\
\hline WindFarm-1 & $8.35 \mathrm{~m} / \mathrm{s}(9.43 \mathrm{~m} / \mathrm{s}, 2.38)$ & $250^{\circ}\left(\mathrm{std}=78.5^{\circ}\right)$ \\
WindFarm-2 & 8 & $\mathrm{~m} / \mathrm{s}(9.01 \mathrm{~m} / \mathrm{s}, 2.25)$ & $210^{\circ}\left(\mathrm{std}=100^{\circ}\right)$ \\
WindFarm-3 & $7.6 \mathrm{~m} / \mathrm{s}(8.54 \mathrm{~m} / \mathrm{s}, 2.57)$ & $220^{\circ}\left(\mathrm{std}=80^{\circ}\right)$ \\
WindFarm-4 & $7.6 \mathrm{~m} / \mathrm{s}(8.60 \mathrm{~m} / \mathrm{s}, 2.48)$ & $202^{\circ}\left(\mathrm{std}=98^{\circ}\right)$ \\
\hline
\end{tabular}


shape and scale parameters of the fitted Weibull distribution and, mean and standard deviation of wind directions of each wind farm. A total of 366 days (105408 samples), from November $1^{\text {st }} 2011$ until October $31^{\text {st }} 2012$, conform the recording period.

NWP-based forecasts from Environment Canada (EC) were available for all sites, offering wind speed estimates at heights $H=\{10,40,120,216\} \mathrm{m}$. EC delivers a set of forecasts values four times a day. The sets include forecasts for the next 3 to 48 hours with a 15 minutes time step. Linear interpolation is used to estimate wind speed at the hub height $(80 \mathrm{~m})$.

WindFarm-1 is presented in this paper to demonstrate the effectiveness of the proposed technique. Fig. 1 shows plots of observed values and monthly averages of wind speed along with a windrose diagram of wind directions. It is worth noting that prevailing and strong winds mainly blow from West and North-West with slower winds occurring in the summer. Noon and nights exhibit slower wind speeds than early morning and evening, which is reported to affect the accuracy of some NWP based predictors [18]. These elements will help selecting input features for one of the forecasting techniques to be used in the proposed fusion.

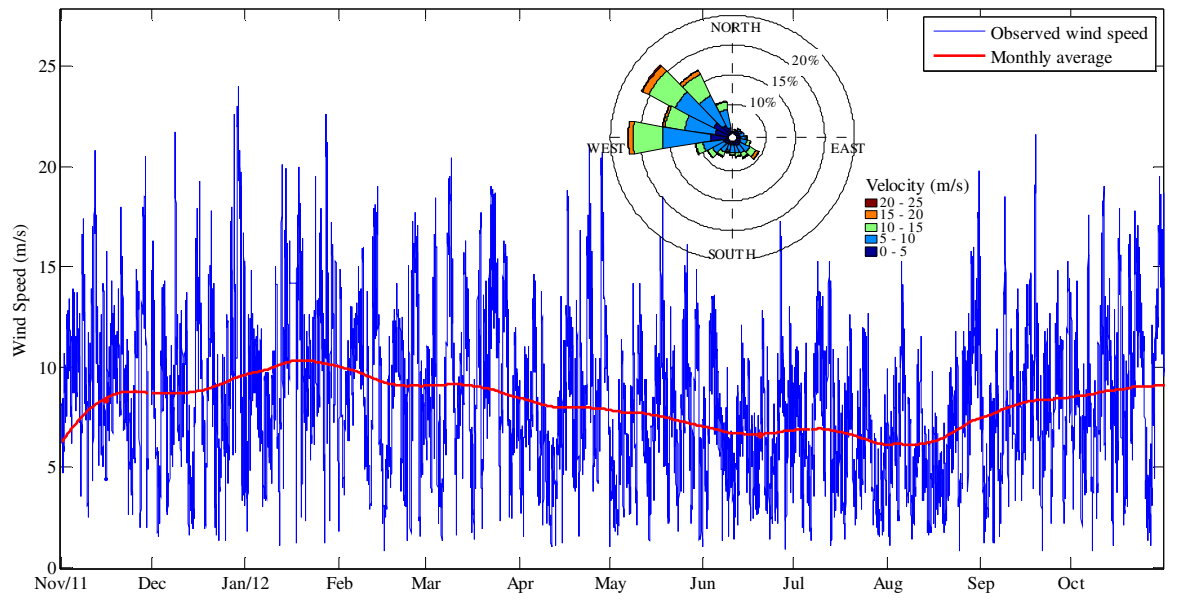

Fig. 1. Measured wind speeds and main wind directions at WindFarm-1

\section{Performance Evaluation}

An effective comparison of forecasting methods is not easily achieved as there are various performance criteria used by researchers [1,9]. Recently, partners of the ANEMOS project have proposed a set of recommendations to establish a common ground for comparison $[9,10]$. Some suggested error criteria include:

- mean absolute error (MAE),

$$
M A E=\frac{1}{N} \sum_{i=1}^{N}\left|\hat{a}_{i}-a_{i}\right|
$$


- root-mean-squared error (RMSE),

$$
R M S E=\sqrt{\frac{1}{N} \sum_{i=1}^{N}\left(\hat{a}_{i}-a_{i}\right)^{2}}
$$

- mean absolute percentage error (MAPE).

$$
M A P E=\frac{100}{N} \sum_{i=1}^{N} \frac{\left|\hat{a}_{i}-a_{i}\right|}{\left|a_{i}\right|}
$$

$\mathrm{N}$ is the size of the sample used to calculate the measure, and $\hat{a}_{i}=\hat{a}_{i}(t+k \mid t)$ and $a_{i}=a_{i}(t+k)$ are the forecasted and the actual values respectively. MAE and RMSE express global errors in terms of wind speed; but RMSE accentuates the effect of larger errors. MAPE is a dimensionless, relative measure of global error. Its singularity problem is avoided here by ensuring the exclusion of zero-valued speeds, which have a very low probability of occurrence.

The recommendations also emphasize to build the models and validate them by using cross-validation. We reserved a set of data (15\%) to test model predictions. Training (70\%) and validation (15\%) sets were used to create models and to avoid model to adapt too closely to the training sample (overfitting), respectively. Data for each set was randomly chosen in order to have training samples from any time of the year.

Finally, comparing farms with differently variable time series should use a skill score $\mathrm{SS}_{\gamma}^{\text {ref }}(\mathrm{k})$. This is an objective indicator of the improvement a forecasting model has over a reference (ref) model at horizon $\mathrm{k}$, using some metric $\gamma[2]$.

$$
S S_{\gamma}^{r e f}(k)=\frac{\gamma^{r e f}(k)-\gamma(k)}{\gamma^{r e f}(k)} \times 100 \%
$$

\section{Individual Wind Speed Forecast Techniques}

\subsection{Persistence or Naïve Model}

This is a simplistic model where its k-step-ahead forecast is expressed as:

$$
\hat{a}(t+k \mid t)=a(t),
$$

i.e. future predicted values will be the same as the last observed value. Its accuracy is very high at the shortest horizons and cannot be easily outperformed by other, more complex models. For this reason it is almost universally used as a benchmark for short-term forecasting of less than six hours. Using persistence in combination with other models ensures high precision and simplicity at the shortest time leads.

\subsection{The Artificial Neural Network Approach}

Artificial neural networks are amongst the most successful machine learning approaches to short-term wind speed time-series forecasting. Improvement over other machine learning and statistical models has been extensively reported in the literature. Although several network architectures have been proposed in forecasting applications, the feed-forward network is one of the most popular [10-13]. 
In this study, we used a feed-forward multilayer perceptron (MLP) neural network architecture using the Levenberg-Marquardt backpropagation (LM) training algorithm. The selected training algorithm encompasses the Newton and gradient descent methods being one of the fastest algorithms. Mean squared errors (MSE) are minimized, which is appropriate for wind speed forecasting where prediction errors are assumed to have a Gaussian distribution [6]. For a multi-step-ahead prediction several approaches might be followed namely iterative, multi-model, or single-model-multivariate forecasting. Iterative forecasting predicts successive look-ahead times by aggregating previous forecasts to the input series. This solution might lead to decreasing accuracy as lookahead time increases, due to accumulation of errors [12]. Single-model-multivariate forecasting uses output layers with as many neurons as the number of look-ahead times, leading to very complex systems. Finally we chose the multi-model forecasting. This approach builds a set of models for each time step; the individual networks are small, faster to train and less likely to be overfitted. A single-neuron linear output layer at each $k^{\text {th }} \mathrm{NN}$ outputs $\hat{\mathrm{a}}_{\mathrm{i}}(\mathrm{t}+\mathrm{k} \mid \mathrm{t})$ wind speed estimates.

Exploratory analysis of the measured weather variables and a bootstrap aggregation of regression trees helped selecting the set of input features by providing measures of variable importance [19]. A final selection of eleven features includes lagged values of wind speed, wind direction, and temperature averages over the last three days prior to the forecasting date and the hour of the day. Having chosen the number of inputs, we addressed the problem of identifying an appropriate network topology. Two hidden layers with hyperbolic tangent sigmoid transfer functions with 20 neurons each resulted as the best configuration after running a set of trials. Fig. 2 depicts a plot of the global MAPE values obtained with these NNs.
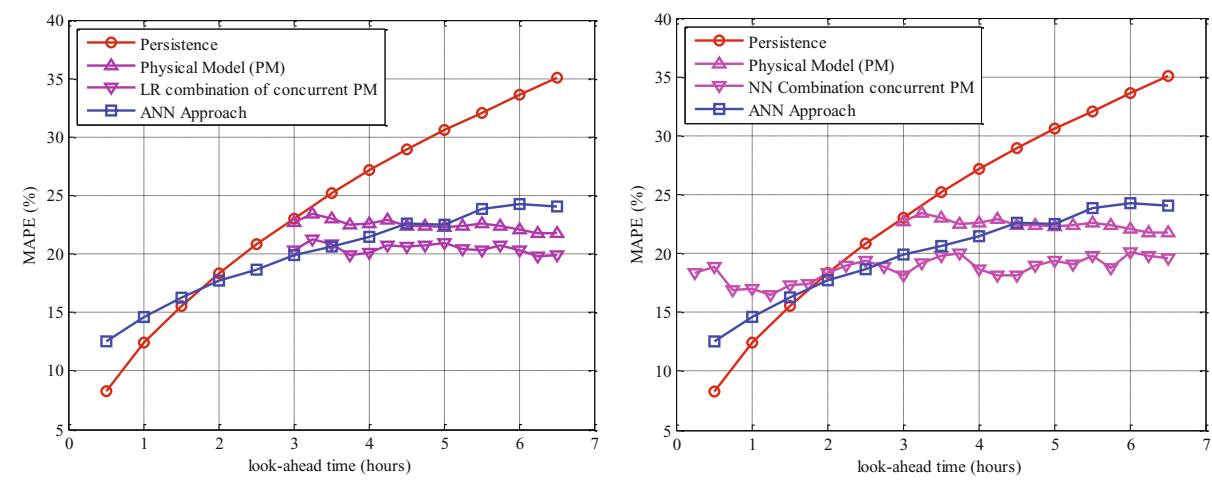

Fig. 2. Performance of individual forecasters

\subsection{Forecast from a Physical Model Based on NWP}

In order to include NWP-based forecasts as an aggregated approach in the final forecast, we decided to combine concurrent sets of EC's forecasts. EC's multi-step-ahead forecasts $\mathrm{F}(\mathrm{T})$ are delivered every $\mathrm{D}=6$ hours, generating an overlap between current 
$\mathrm{F}(\mathrm{T})$ and previous $\mathrm{F}(\mathrm{T}-\mathrm{nD})$ forecasts. These overlaps can be used to extend the time span of the forecasts below 3 hours and to further improve the predictor's performance; particularly if concurrent forecasts can be properly combined.

When concurrent forecasts $\hat{a}_{i}$ are comparable, the combination can be reduced to simple average of competing forecasts. More complex combinations can also be exploited using more complex mixing functions. We tested several of these methods including simple averaging of concurrent forecasts, linear regression (LR), and NN. Simple averaging assigns equal weights to all, present and past, forecasts and does not eliminate any bias of the original forecasts. Regression and $\mathrm{NN}$ based approaches assign weights that act as "forgetting" factors indicating the influence of each previous forecast in the combined forecast. Fig. 2 graphically shows the improvement attained by linear regression and NN-based combinations. This figure also shows average MAPE performance for the other two independent forecasters. Individual models do not resemble competing models' forecasts at all k-step-ahead times. As expected, persistence performs best for look-ahead times below two hours. The relative performance between the NN approach and the combination of concurrent NWPbased forecasts depends on the combination approach employed.

\section{Fusion Forecast Method}

Independent forecasters are combined to generate a final forecast. The proposed combination should generate multi-step-ahead, short-term forecasts from five minutes, up to 6:00 hours ahead. The time step between time leads is set to five minutes. As aforesaid, although independent forecasters can be further improved, we decided to assign the task of performance improvement to the combination method. For this reason we selected simple averaging of concurrent NWP-based forecasts over NN or LR approaches as an independent model. This decision makes the overall system simpler.

Artificial neural networks have been successfully applied for forecast combination [14-16]. In this paper we investigated a NN architecture that optimizes the assigned

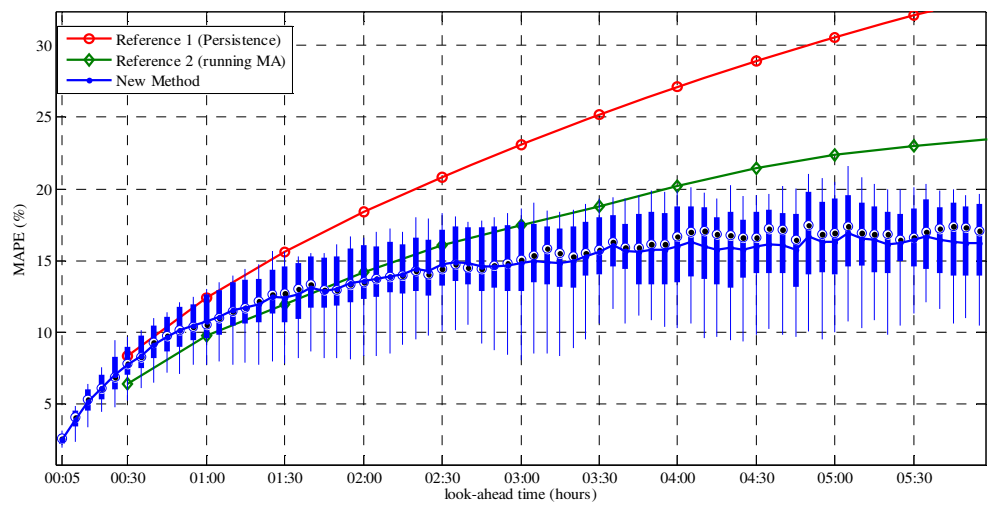

Fig. 3. Performance of the proposed hybrid method 
weights to each forecaster at each time lead. Again, we decided to build a bank of simple neural networks, corresponding with our look-ahead times. Each network was designed as feed-forward with one hidden layer of 10 neurons and a hyperbolic tangent sigmoid transfer function. The output layer had only one neuron and a linear transfer function to produce one-step-ahead forecasts. The LM algorithm was used for training with the MSE distortion measure as the performance function.

Fig. 3 illustrates the monthly MAPEs for every prediction horizon and mean MAPEs are available for two reference models, namely persistence (Pers) and the moving average (MA) reference recommended by several authors [9, 10]. Box plots are also displayed for the proposed fusion method. From 10 minutes ahead, the proposed method is increasingly better than persistence. Similar behavior stands when comparing with the MA reference after 1:45 hours. The box plots show increment of error dispersion with increased horizons. The method also shows consistency after three hours ahead as error dispersion does not increase.

Table 2 shows some performance metric values at different horizons. It also shows how the proposed method holds significant gains over reference models as look-ahead times increase. Skill scores (SS) calculated using different error criteria show from $35 \%$ to more than $50 \%$ improvement over persistence, and from $16 \%$ to above $30 \%$ improvement over the recommended MA reference.

Table 2. Performance metrics and skill scores at different horizons

\begin{tabular}{|c|c|c|c|c|c|c|c|c|c|}
\hline \multirow{2}{*}{ Horizon } & \multirow{2}{*}{$\begin{array}{c}M A E \\
(m / s)\end{array}$} & \multicolumn{2}{|c|}{$S S_{M A E}(\%)$} & \multirow{2}{*}{$\begin{array}{c}R M S E \\
(m / s)\end{array}$} & \multicolumn{2}{|c|}{$S S_{R M S E}(\%)$} & \multirow{2}{*}{$\begin{array}{c}M A P E \\
(\%)\end{array}$} & \multicolumn{2}{|c|}{$S S_{M A P E}(\%)$} \\
\hline & & Pers & MA & & Pers & MA & & Pers & MA \\
\hline $0: 30$ & 0.543 & 9.46 & -8.31 & 0.736 & 11.09 & -7.76 & 7.75 & 6.89 & -9.47 \\
\hline 1:00 & 0.738 & 15.86 & -8.27 & 0.978 & 17.58 & -6.73 & 10.78 & 13.05 & -10.08 \\
\hline 2:00 & 0.915 & 28.35 & 8.08 & 1.200 & 29.53 & 8.44 & 13.61 & 25.90 & 6.20 \\
\hline 3:00 & 0.984 & 38.86 & 17.91 & 1.266 & 40.50 & 19.30 & 14.79 & 35.87 & 15.59 \\
\hline 4:00 & 1.063 & 42.95 & 21.56 & 1.356 & 44.45 & 23.13 & 15.99 & 41.09 & 21.43 \\
\hline 5:00 & 1.077 & 48.37 & 28.02 & 1.381 & 49.20 & 28.66 & 16.31 & 46.61 & 28.61 \\
\hline 6:00 & 1.082 & 52.75 & 31.25 & 1.386 & 53.54 & 32.05 & 16.24 & 51.69 & 32.61 \\
\hline
\end{tabular}

\section{Concluding Remarks}

Wind speed forecasting is today an important research topic for a continued increase of wind power penetration into the global power market. In this paper, we presented an effective fusion-based wind speed prediction method that non-linearly combines the results of three different forecasters. Experimental results show that a Neural Network combination of forecasts improves performance over individual methods used in the combination. Error reductions up to more than $50 \%$ with respect to persistence and more than $30 \%$ over the recommended MA reference predictors are obtained with different error measures. Even for horizons where only one independent method prevails over the rest, the fusion approach improves performance.

Although not explicitly shown in the paper, the application of this methodology to the other sites listed in Table 1 gave similar results. 
We agree with other authors [13] in that better models would be obtained increasing the dataset to more than one year. Adaptation of current models as more data become available is another alternative for improving models.

Results from this paper can be extended in a number of directions. First, improved independent models could be used. This has the advantage of enabling the fusion approach to offer a rather good prediction when the other methods fail. Secondly, the adaptation to other wind farms should also be investigated without explicitly training the combination forecast to each wind farm. Finally, including probabilistic error bounds instead of point forecasting should also be investigated.

\section{References}

1. Zhu, X., Genton, M.G.: Short-Term Wind Speed Forecasting for Power System Operations. International Statistical Review 80, 2-23 (2012)

2. Foley, A.M., Leahy, P.G., Marvuglia, A., McKeogh, E.J.: Current methods and advances in forecasting of wind power generation. Renewable Energy 37, 1-8 (2012)

3. Stathopoulos, C., Kaperoni, A., Galanis, G., Kallos, G.: Wind power prediction based on numerical and statistical models. Journal of Wind Engineering and Industrial Aerodynamics 112, 25-38 (2013)

4. Wang, X., Guo, P., Huang, X.: A Review of Wind Power Forecasting Models. Energy Procedia 12, 770-778 (2011)

5. Soman, S.S., Zareipour, H., Malik, O., Mandal, P.: A review of wind power and wind speed forecasting methods with different time horizons. In: North American Power Symposium (NAPS), pp. 1-8 (2010)

6. Lange, M., Focken, U.: New developments in wind energy forecasting. In: 2008 IEEE Power and Energy Society General Meeting - Conversion and Delivery of Electrical Energy in the 21st Century, pp. 1-8 (2008)

7. Costa, A., Crespo, A., Navarro, J., Lizcano, G., Madsen, H., Feitosa, E.: A review on the young history of the wind power short-term prediction. Renewable and Sustainable Energy Reviews 12, 1725-1744 (2008)

8. Ernst, B., Oakleaf, B., Ahlstrom, M.L., Lange, M., Moehrlen, C., Lange, B., Focken, U., Rohrig, K.: Predicting the Wind. IEEE Power and Energy Magazine 5, 78-89 (2007)

9. Madsen, H., Pinson, P., Kariniotakis, G., Nielsen, H.A., Nielsen, T.: Standardizing the Performance Evaluation of ShortTerm Wind Power Prediction Models. Wind Engineering 29, 475-489 (2005)

10. Giebel, G., Brownsword, R., Kariniotakis, G., Denhard, M., Draxl, C.: The state-of-the-art in short-term prediction of wind power: A literature overview. ANEMOS. plus (2011)

11. Salcedo-Sanz, S., Pérez-Bellido, Á.M., Ortiz-García, E.G., Portilla-Figueras, A., Prieto, L., Correoso, F.: Accurate short-term wind speed prediction by exploiting diversity in input data using banks of artificial neural networks. Neurocomputing 72, 1336-1341 (2009)

12. Kusiak, A., Zheng, H., Song, Z.: Short-term prediction of wind farm power: a data mining approach. IEEE Transactions on Energy Conversion 24, 125-136 (2009)

13. Li, G., Shi, J.: On comparing three artificial neural networks for wind speed forecasting. Applied Energy 87, 2313-2320 (2010)

14. Donaldson, R.G., Kamstra, M.: Forecast combining with neural networks. Journal of Forecasting 15, 49-61 (1996) 
15. Palit, A.K., Popovic, D.: Nonlinear combination of forecasts using artificial neural network, fuzzy logic and neuro-fuzzy approaches. In: The Ninth IEEE International Conference on Fuzzy Systems, FUZZ IEEE 2000, vol. 2, pp. 566-571 (2000)

16. Aladag, C.H., Egrioglu, E., Yolcu, U.: Forecast Combination by Using Artificial Neural Networks. Neural Process. Lett. 32, 9156:269-9156:276 (2010)

17. Timmermann, A.: Chapter 4 Forecast Combinations. In: Elliott, G., Granger, C.W.J., Timmermann, A. (eds.) Handbook of Economic Forecasting, pp. 135-196. Elsevier (2006)

18. Lange, M.: On the Uncertainty of Wind Power Predictions-Analysis of the Forecast Accuracy and Statistical Distribution of Errors. Journal of Solar Energy Engineering 127, 177 (2005)

19. Strobl, C., Malley, J., Tutz, G.: An Introduction to Recursive Partitioning: Rationale, Application and Characteristics of Classification and Regression Trees, Bagging and Random Forests. Psychol. Methods 14, 323-348 (2009) 